\title{
Open Software Experiment System based on Single Chip Microcomputer
}

\author{
Xingshan $\mathrm{Li}^{1,}$, , Jian $\mathrm{Lu}^{2}$ \\ ${ }^{1}$ Luohe medical college, Luohe, 462000, China \\ ${ }^{2} \mathrm{Henan}$ industrial vocational and technical college, Nanyang, 473000, China \\ aEmail:604141388@qq.com
}

Keywords: Single Chip Microcomputer, FX-A Full Development Board, Serial Memory Module, Liquid Crystal Display Interface

\begin{abstract}
The paper expounds the design scheme of experimental platform, the serial storage module, LCD display interface, buzzer driving circuit and extend experimental performance of software experimental area was introduced in detail, put forward the innovative design for imaging electronics course teaching experimental device, the experimental system is tested to meet the design requirements, the results of the project to take a most intuitive and feasible the way to replace the existing teaching equipment, which can be used after learned, can be used at any time and can meet the requirements of after-school students' autonomous experiments.
\end{abstract}

\section{Introduction}

How to improve the practice ability of medical college students, it has always been a problem in the teaching work of teachers. In my school, the number of students rapid increase in recent years, Laboratories and experimental and practical training equipment resources shortage, which has affected normal teaching of the teacher. So, it is particularly urgent to develop a set of experimental platform which can meet the needs of teaching and low cost. Project team members design a set of for video electronics basic course and specialized course experiment platform solutions to solve the problems encountered in teaching [1]. Project team members based on the actual situation of the school, put forward the development of practical platform for the development of performance indicators are as follows:

1. The practice platform is easy to operate, easy for students to use in experiments, and make all kinds of medical electronic circuits.

2. Can cooperate with the relevant instruments to complete all experimental and training projects of the medical analog electronics and digital electronic technology.

3. Can finish the experiment training project of software and hardware in single chip microcomputer technology and interface technology teaching.

4. Practical platform can effectively help students to fully grasp the relevant technology, with the experimental functions of full analysis and development.

5. The practice platform has the function extension, can carry on the promotion and the expansion along with the curriculum to be convenient to carry on the promotion and the expansion.

6. The appearance of the practical platform is beautiful, generous, easy to carry and has unique characteristics in the market.

7. Practice platform cost control in less than 300 yuan, easy mass production, can be widely used.

The purpose of project development is to make every student use the platform to improve their practical ability. Although the cost is low, but to meet the various needs of teaching is the focus of our project. "Medical imaging single-chip computer application technology" course as a project service target, if the project can solve this kind of professional course, then the experiment platform must be used in analog electronics, digital electronics, circuit basis, practice of the course. According to this idea, the project team members and detailed division of labor, respectively for appearance, experimental development board, the system programmer adapter, function module, 
programming software technology research. After the practical platform development, we named it "open electronics experiment system". The suit is mainly includes five parts, respectively is "FX - A complete analysis development board", "programming adapter", "FX - pro programming software", "fusion programmer", "fusion function module[2] ".

\section{Software experimentation area performance}

Development board software experimentation area is based on the students to learn microcontroller technology experimental requirements to develop a multi-function circuit board, able to complete as many as more than ten kinds of experiments and portfolio projects, and with the ISP online programming function [3]. Multifunctional circuit board circuit system including power supply module, SCM smallest system design, water lamp display module, digital tube dynamic display, serial interface, the key input, the serial storage module, LCD display interface, buzzer driving circuit, parts of the circuit board are now described as follows:

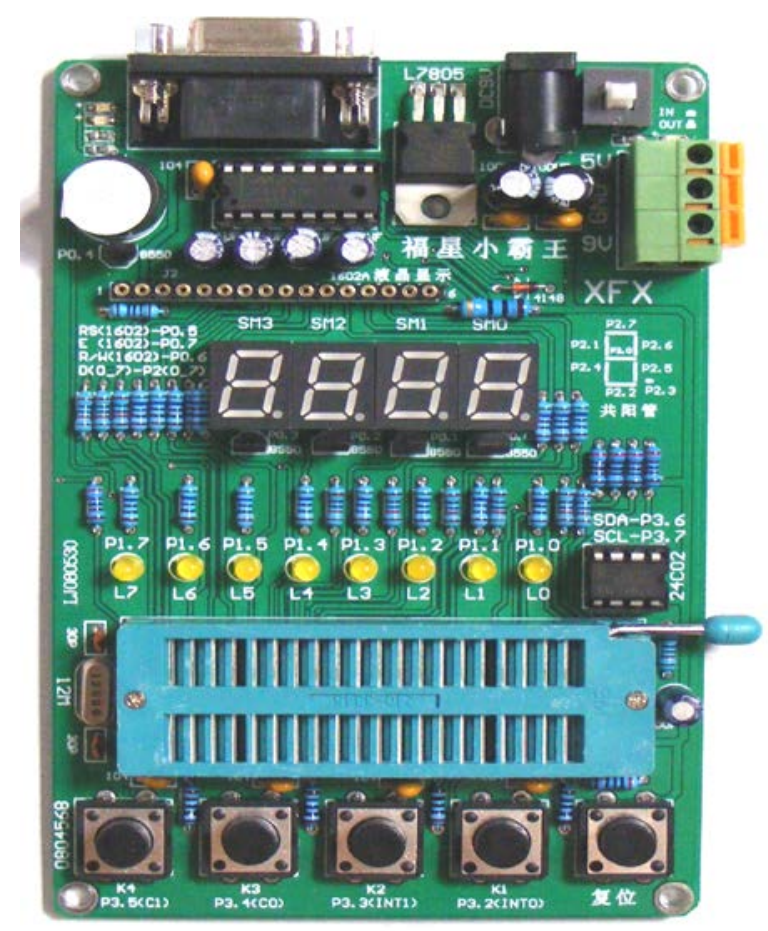

Fig1 Software experimentation area

\section{Serial memory module.}

In single-chip applications, often use some data needs to be saved for a long time, the traditional method of cost is higher, the circuit is more complex, in recent years, nonvolatile memory technology development is rapid, EEPROM is one of the 24 series used widely in the block is the EEPROM, this series of chip only 8 pins, using 2 wire system I2C interface. For the convenience of learning the use of the chip, the socket can be inserted into AT2401, AT2402 chip experiments. The test circuit board line as shown in Figure 2, where SDA is the data bus in the I2C bus, SCL clock line in I2C, and the WP pin is write protected line of the chip, the data line I2C (SDA) is an open drain output, between the VCC pin and connected with a pull-up resistor. 


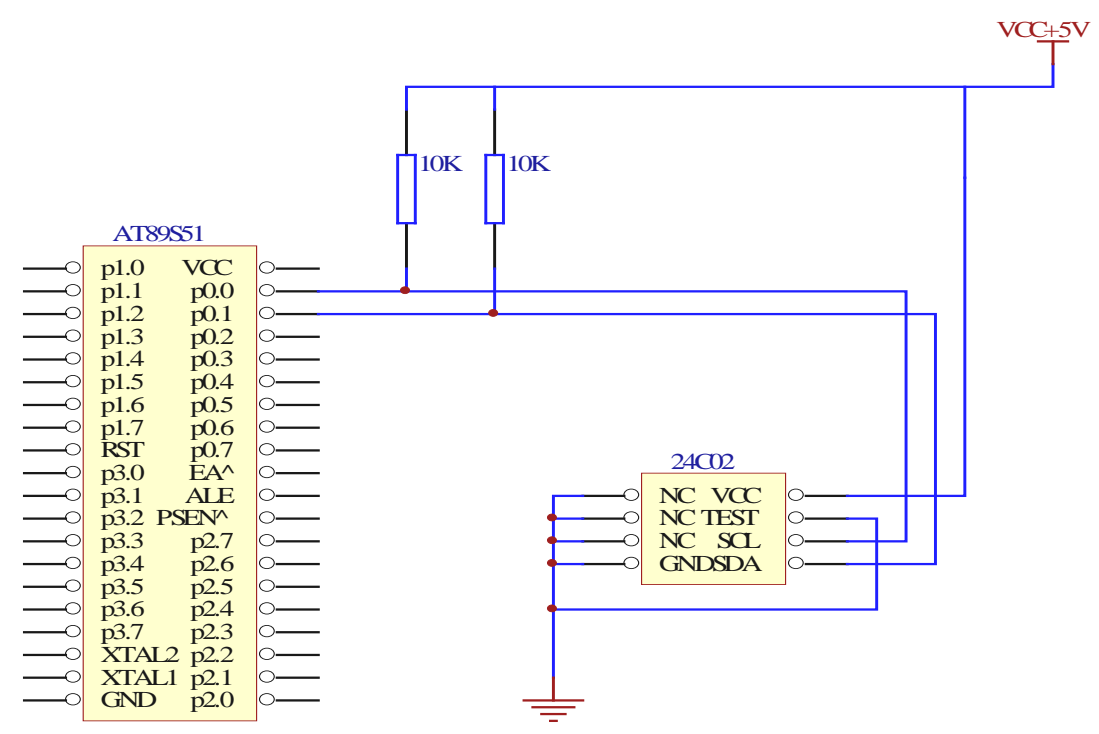

Fig 2 Serial memory interface

\section{Liquid crystal display interface.}

In order to use the user to master the use of liquid crystal display, special design standard 16 foot interface. 1602A character type liquid crystal direct insertion can be convenient for users to use. The LCD with backlight, bring their own character library. Display 2 lines each line of 16 characters, the standard 16 pin interface. As shown in figure 3, 8 bits of data a P2 port, control termination P0 mouth.

\section{Buzzer driving circuit.}

The output of the MCU I/O port of low level driver ability better than high level, but also at guarantee the logical levels will only be about $5 \mathrm{ma}$, don't need to make sure that the logical levels

(For example, when driving LED can have more than $10 \mathrm{~mA}$ ). Therefore, if you want to drive the high power devices: (1) Should be subject to low level; (2) External power driving device[4]. Buzzer to drive current is bigger, according to the above consideration, the experimental plate adopts the buzzer driver circuit as shown in figure 4, with the main chip P0.4 as a source of sound, the triode drive a passive generator or buzzer sound. The circuit structure is simple and convenient.

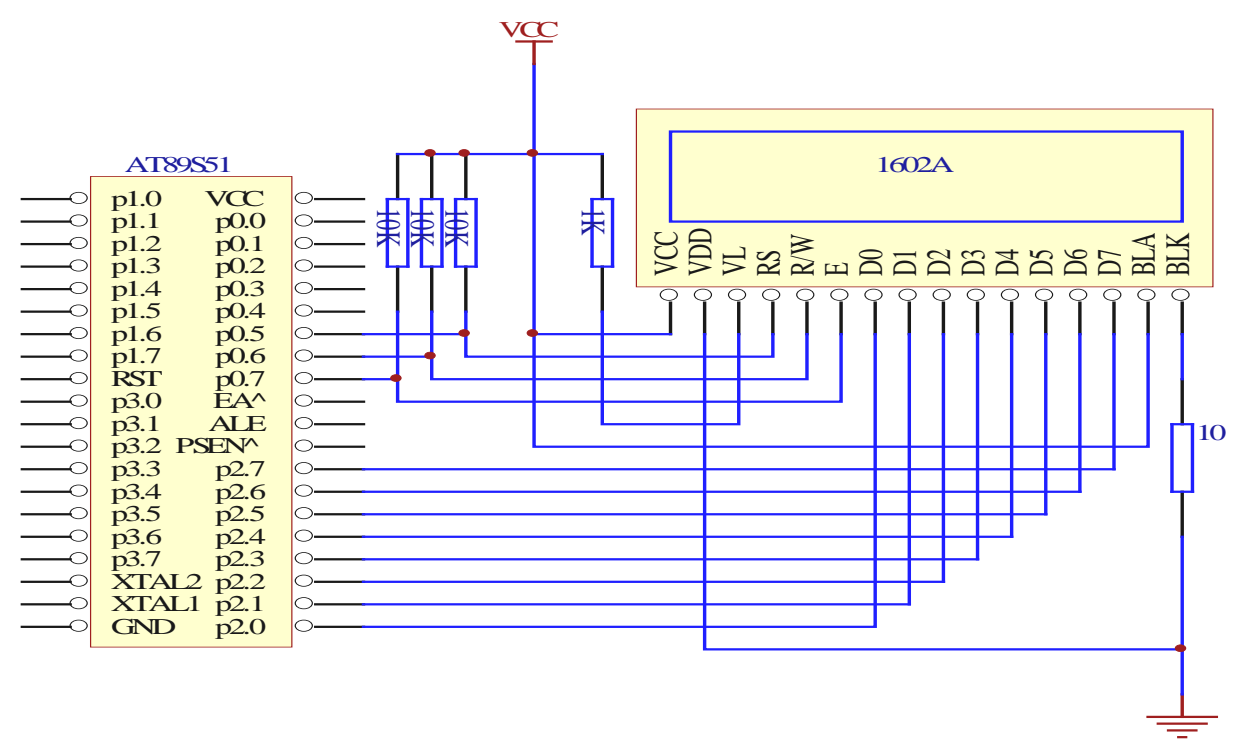

Fig3 Liquid crystal display interface 


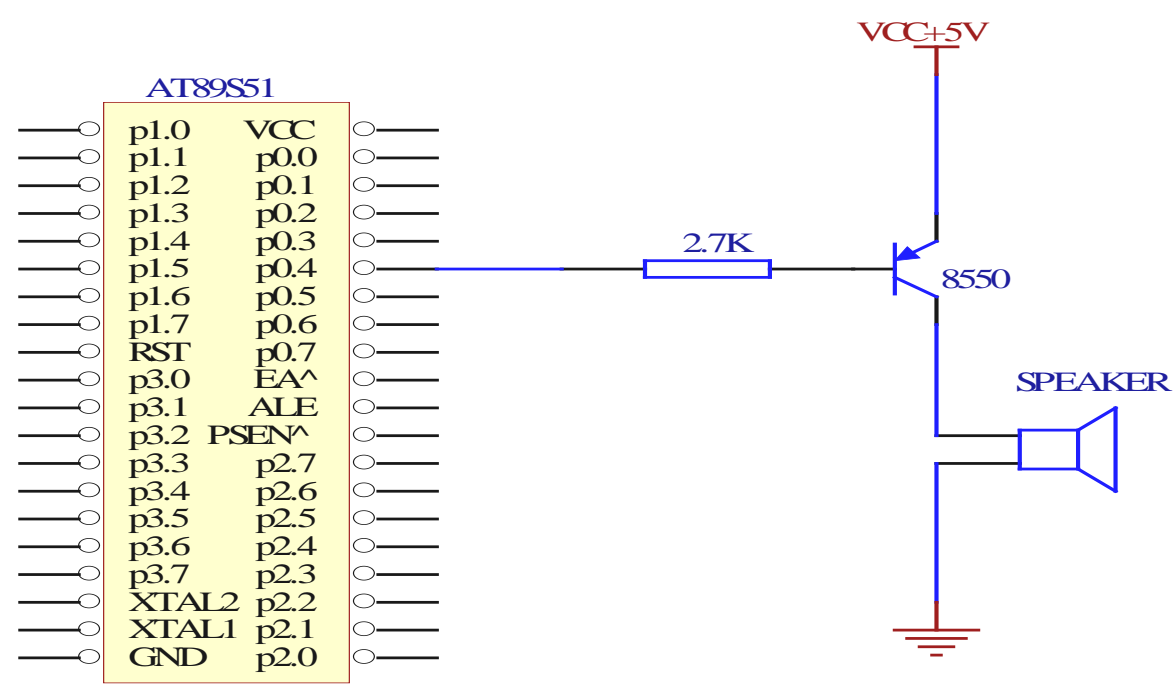

Fig 4 Buzzer driving circuit

\section{FX-A extended experimentation area performance}

FX - A hardware experimental area (extension) through three low cost and reliable performance of the bread plate, and Jacques force board, beginners can go to the market to buy, then according to the characteristics of the bread plate and required to install on the top of the Jacques force board.

For starters, the matters needing attention and instructions about the bread plate were added between Jacques and the bread plate for the beginners quickly to fit extend board.

FX - A development board is mainly lets the student observe the experiment phenomenon, master the programming thought and method, the hardware experimental area users can independently complete the following experiment: Water lights, digital tube display, query, external interrupt, timer application independent buttons, timer interrupt, timer interrupt, serial port communication, serial port interrupt application, dynamic display of digital tube, 24, speaking, reading and writing, buzzer playing our fleet memory, LCD, according to a 1602 a and can also use fusion series of module further expansion[5].

Beginners through experimental area of hardware connection circuit, electric test, experimental contrast observed experimental phenomena in FX - A software, can easily find out the hardware connection issue. Through the study, not only improve yourself interested in SCM, the most simple operation to understand SCM, then can be done alone by extending plate above experiment, inspire their study of single chip microcomputer internal structure. In the end, we can face the actual problems in the life, carry on the experiment in the hardware experimentation area, analyze the problem, and finally solve the problem.

Here are the instructions on the use of the bread plate as well as the matters needing attention: The bread plate appearance and internal structure as shown in figure 5 , the bread plate consists of two parts, the upper portion is generally made up of a line or two lines of jack narrow strip, no electrical connectivity between line and line. Every five columns jack as a group, usually have 10 or 11 group on your bread plate. For structure of 10 groups, the left three groups internal electrical connectivity, internal electrical connectivity among four groups, on the right side of the three groups of internal electrical connected, but the left three groups, among four groups and between the three groups on the right is not connected. For structures of 11 groups, the internal electrical were connected in 4 groups on the left side, the three groups of middle internal electrical connectivity, on the right side of the four groups of internal electrical connectivity, the middle of the 3 groups of internal electrical connectivity, the 4 groups on the right side of the internal electrical connectivity, but the 4 groups on the left, the 3 groups of middle and the right side of the 4 groups are not connected. If you need to use the connection, you must cross the wire between the two. The following part is made up of an isolation grooves and up and down the 5 rows of jacks. In 
the same column 5 socket is connected to each other, and grooves between column and column top and bottom part is disconnected.

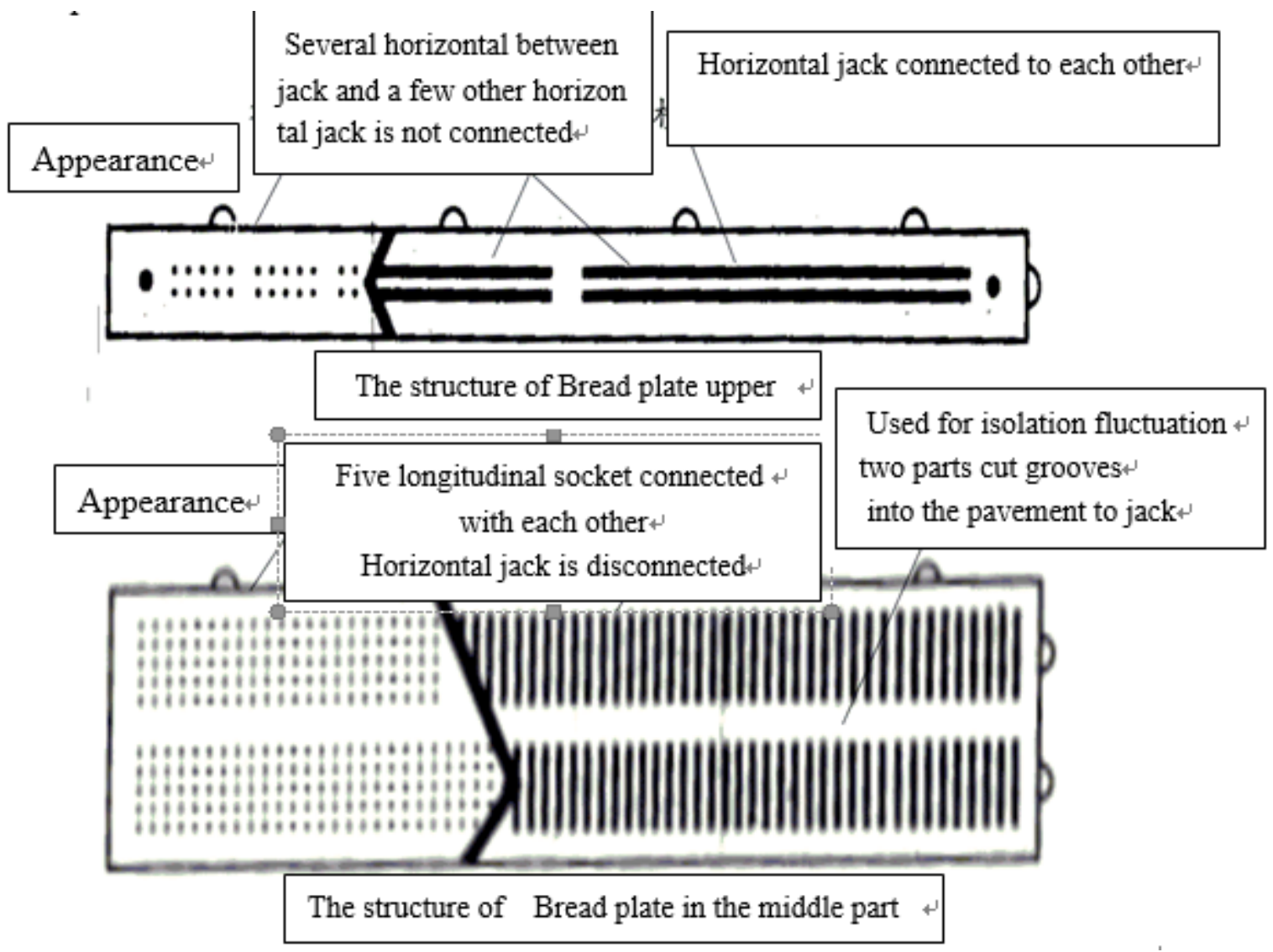

Fig 5 Bread plate

In the specific use of time, usually two narrow a wide use at the same time, the first line of the two narrow strip in general and the ground wire connection, the second line and the power connected. Due to the integrated power module in the above general, ground wire is in commonly the following. Such a layout will help the power of the integrated block feet and connected to the second line above narrow strip, Ground under the feet and narrow connected to the first line of the article, Reduce the number of wire length and jumper. The middle width strip is used for connecting the circuit, and the groove is not communicated with the upper and the lower part of the groove, so the integrated block is generally inserted in the groove.

\section{Summary}

The circuit is tested, and the test parameters of the circuit board meet the design requirements.

\section{Acknowledgement}

In this paper, the research was sponsored by the education technology and equipment and practice education research project topics in Henan Province In 2015 (Project No. GZS112), and by the Nature Science Foundation of Luohe Medical College (2013-S-LMC27 ). 


\section{References}

[1] Xinyu Zhu. Exploitation and application of electronic design automation experiment system[D] : [A master's degree thesis]. Beijing: Beijing university of posts and telecommunications, 2012.

[2] Guoyan Duan, Research and development of electronic technology virtual experiment system [D] : [A master's degree thesis]. Chongqing: Southwest jiaotong university, 2013

[3] Lei Su; Development and application of the separated electrical experiment device [J].Electronic design engineering, 2011, 19 (9) :138 -140.

[4] Chunyu Cheng, Zhenyu Wu, Yanan Wu,et al.Development of analog electronic technology experiment platform for hierarchical teaching mode[J].Laboratory science, 2014,17 (5) :34 -37.

[5] Shisheng Yan, Chengyao Zhong, Liefeng Wang. Research and development of new analog electronics technology experimental device [J]. Journal of Hainan Normal University (Natural Science), 2012, 25 (3):283 -285. 